\title{
Contribution à la dépollution des eaux usées de textile par électrocoagulation et par adsorption sur des composés à base de fer et d'aluminium
}

\author{
Fatiha ZIDANE ${ }^{1 *}$, Adil RHAZZAR ${ }^{1}$, Jean-François BLAIS ${ }^{2}$, Kamal AYOUBI ${ }^{1}$, \\ Jalila BENSAID ${ }^{1}$, Soumia EL BASRI ${ }^{1}$, Noureddine KABA ${ }^{1}$, Qassid FAKHREDDINE ${ }^{1}$ \\ et Brahim LEKHLIF ${ }^{3}$ \\ ${ }^{1}$ Laboratoire Science de l'Eau et d'Environnement, Département de Chimie, Faculté des Sciences Ain Chock, \\ Université Hassan II, Km 8, BP 5366, Maarif, Casablanca, Maroc. \\ ${ }^{2}$ Institut National de la Recherche Scientifique (INRS-ETE), Université de Québec, 490, Rue de la couronne, \\ QC JIK 9A9, Québec, Canada. \\ ${ }^{3}$ Ecole Hassania des Travaux Public, Km 7, Route d'El Jadida, Casablanca, Maroc. \\ *Auteur correspondant, E-mail : zidanefatiha@hotmail.com, Tel: +212661330342
}

\section{RESUME}

Les ressources hydriques au monde sont rares et la demande en eau connait une croissance continue en liaison avec l'évolution démographique et les activités consommatrices en eau, notamment les industries de textiles se voient dans l'obligation de recycler les eaux résiduaires et en particulier celles colorées. Dans ce travail, nous nous sommes intéressés à l'étude de l'élimination des matières organiques et colorantes de deux rejets provenant des industries de textile, un de teinture du tissu de polyester à $\mathrm{pH}$ acide et l'autre de teinture du tissu de coton à $\mathrm{pH}$ basique. Ces rejets ont été traités de deux manières. La première est par électrocoagulation en utilisant des plaques de fer et/ou d'aluminium. La deuxième est par adsorption sur des composés synthétiques à base de fer et d'aluminium préparés par électrocoagulation. Dans le cas du traitement par le procédé d'électrocoagulation, nous avons constaté que le rendement d'élimination en demande chimique en oxygène (DCO) du rejet de polyester atteint un rendement de DCO de $60 \%$ pour un temps de 7 min de réaction. Pour le rejet de coton, le rendement d'élimination, par les plaques de fer/aluminium et l'élimination des matières colorantes, atteint une valeur de $45 \%$ en terme de DCO, et ceci en utilisant des plaques d'aluminium seul et de fer/aluminium pour un temps de 12 et 15 min respectivement. Dans le cas du traitement par ajout des coagulants synthétiques préparés au laboratoire, nous pouvons observer que le meilleur rendement d'élimination en DCO du rejet de polyester est obtenu pour une valeur de $48 \%$, pour la faible granulométrie avec une concentration de $5 \mathrm{~g} / \mathrm{l}$ du composé à base de fer /aluminium. Le rendement d'élimination en DCO du rejet de coton augmente jusqu'à une valeur de $60 \%$ avec une concentration de $5 \mathrm{~g} / \mathrm{l}$ de coagulants appliqués à base d'aluminium seul. Les résultats de la dépollution de ces rejets, ont montré que le rendement d'élimination des matières organiques et colorantes par le procédé d'électrocoagulation est important, et la durée de traitement est courte, mais l'inconvénient de ce procédé c'est la saleté des plaques après chaque utilisation et la fabrication d'une grande quantité des boues par rapport à l'adsorption sur des composés à base de fer /aluminium où on utilise des poudres peu solubles et stables avec un bon rendement d'élimination et faibles quantités de fer et d'aluminium dans le surnageant traité.

(C) 2011 International Formulae Group. All rights reserved.

Mots clés : Colorant textile, électrocoagulation, adsorption, dépollution, fer, aluminium.

\section{INTRODUCTION}

La présence de nombreux colorants dans les rejets de l'industrie du textile représente un véritable danger pour l'homme et son environnement (Bell et Buckleyc, 2003), en raison de leur stabilité chimique vis-

() 2011 International Formulae Group. All rights reserved. DOI : http://dx.doi.org/10.4314/ijbcs.v5i4.35 
à-vis des agents oxydants (rayons UV, oxydants chimiques), et leur faible biodégradabilité (Bouras, 2003). Les procédés traditionnels de traitement de l'eau usée, tels que les procédés biologiques donnent des résultats non satisfaisants (Pillre, 2001; Zidane, 2006). Ainsi la principale contrainte d'utilisation de cette méthode provient de la difficulté d'accumuler des bactéries à certains effluents et leur inhibition par certaines composantes chimiques et aussi, la duré de traitement (Alinsafi et al., 2005).Pour cette raison, des nouvelles recherches sur des nouvelles méthodes. Parmi lesquelles: l'électrocoagulation (Carmona et al., 2006 ; Heidmann et Calmano, 2007; Zuo et al., 2008 ; Bensaid, 2009; Bennajah et al., 2009; Zongo et al., 2009), l'adsorption soit, sur l'argile (Meçabib et al., 2006), sur des composés synthétiques à base d'aluminium (Zidane, 2008) et sur le charbon actif (Ben Tahar et al., 2004 ; Baghriche, 2008), mais cette dernière est très coûteuse et non régénérable.

Le procédé d'électrocoagulation crée dans l'eau à épurer des complexes de type $\mathrm{Al}_{2}(\mathrm{OH})^{5+}, \quad \mathrm{Al}(\mathrm{OH})_{2}{ }^{4+}$ ou des hydroxydes $\mathrm{Al}(\mathrm{OH})_{3}, \mathrm{Fe}(\mathrm{OH})_{2}, \mathrm{Fe}(\mathrm{OH})_{3}$, qui jouent le rôle de coagulant en formant des agrégats de particules qui sont décantées après pour avoir une eau claire (Zongo, 2009).

Le présent article est consacré à l'étude de traitement de deux rejets de textiles, et ceci par deux techniques d'épuration : l'électrocoagulation et l'adsorption sur des composés à base de fer et d'aluminium préparés par l'électrocoagulation et comparer les résultats obtenus avec les deux techniques. Ceci nous a incité à donner un aperçu sur l'effectivité de traitement des eaux usées par ces composés préparés, ainsi l'effet de la granulométrie.

\section{MATERIEL ET METHODES}

Les échantillons de textiles, traités par électrocoagulation ou par action des composés synthétiques, préparés par l'électrocoagulation, sont les suivants :

Rejet 1: Rejet de la teinture du tissu de polyester, d'aspect jaune clair caractérisé par un $\mathrm{pH}$ acide de valeur 4,7. Rejet 2 : Rejet de la teinture du tissu en coton de couleur bleue, d'un $\mathrm{pH}$ basique de valeur 11,2.

\section{Traitement par électrocoagulation à l'aide d'un réacteur à deux électrodes}

Le montage d'électrocoagulation est constitué par une cellule électrolytique en pyrex d'un volume de $500 \mathrm{ml}$ de rejet à traiter, munie de deux électrodes en fer ou aluminium de dimensions $15 \mathrm{~cm} \times 7 \mathrm{~cm} \times 0,2$ $\mathrm{mm}$. La distance inter électrodes est de $4 \mathrm{~cm}$. La tension aux bornes des électrodes est fixée par un générateur de courant continu, le potentiel est maintenu constant à $12 \mathrm{~V}$ durant l'expérience. L'homogénéisation de la solution est effectuée à l'aide d'un agitateur magnétique pour empêcher la formation de dépôts sur le fond et les parois de la cellule.

Pendant les essais, des prélèvements ont été effectués à des intervalles de temps choisis pendant toute la durée de chaque essai. Les paramètres suivis sont le $\mathrm{pH}$, la conductivité, la densité optique et la DCO.

Les plaques utilisées pendant le traitement des rejets par électrocoagulation sont :

- Plaque (Al/Al) : Réacteur avec deux plaques d'aluminium.

- Plaque (Fe/Al) : Réacteur avec une plaque de fer et une d'aluminium.

- Plaque (Fe/Fe) : Réacteur avec deux plaques de fer.

Traitement par adsorption sur des composés à base de fer/aluminium

Le deuxième procédé de dépollution utilisé dans cette étude a été effectué par adsorption sur des composés synthétiques à base de fer, d'aluminium et de fer/aluminium préparés par l'électrocoagulation selon la méthode décrite par Zidane et al. Nous avons effectué des expériences en ajoutant des 
masses croissantes de ces composés de deux granulométries différentes $(X<160 \mu \mathrm{m}$, et160 $\mu \mathrm{m}<\mathrm{X}<500 \mu \mathrm{m})$ dans $25 \mathrm{ml}$ de rejet à traiter. Après un temps de contact de 5 min sous agitation magnétique et un temps de décantation de $24 \mathrm{~h}$, des prélèvements du surnageant ont été effectués afin de déterminer le $\mathrm{pH}$, la densité optique, la conductivité et la DCO.

Les coagulants synthétiques utilisés sont désignés comme suit :

- A3: composé à base d'aluminium seul, préparé en utilisant quatre plaques d'aluminium.

- F1 : composé à base de fer seul, préparé en utilisant quatre plaques de fer.

- AF2 : composé à base de mélange fer/aluminium, préparé en utilisant deux plaques de fer et deux plaques d'Aluminium.

\section{RESULTATS}

Traitement du rejet par électrocoagulation Evolution du pH en fonction du temps pendant le traitement

Les Figures 1 et 2 présentent respectivement l'évolution du $\mathrm{pH}$ en fonction du temps pendant la dépollution du rejet de teinture du tissu en polyester et du rejet de teinture du tissu en coton par électrocoagulation.

D'après la Figure 1, le rejet en polyester se trouvant initialement à $\mathrm{pH}$ acide $(\mathrm{pH}=4,7)$, se comporte différemment vis-à-vis des électrodes de $\mathrm{Fe} / \mathrm{Fe}, \mathrm{Al} / \mathrm{Al}$ et $\mathrm{Fe} / \mathrm{Al}$.

Le réacteur à électrodes d'aluminium ( $\mathrm{Al} / \mathrm{Al})$ montre une évolution croissante de $\mathrm{pH}$, alors que les réacteurs $\mathrm{Fe} / \mathrm{Al}$ et $\mathrm{Fe} / \mathrm{Fe}$ présentent jusqu'à 20 minutes une évolution croissante puis décroissante de celui-ci.

On constate par ailleurs que la courbe $\mathrm{du}$ réacteur $\mathrm{Fe} / \mathrm{Al}$ présente une allure en cloche plus prononcée que celle du réacteur $\mathrm{Fe} / \mathrm{Fe}$. Au bout de 20 minutes on atteint un palier de $\mathrm{pH}$ proche de 6 dans le cas de l'aluminium seul, de 9 dans le cas du mélange $\mathrm{Al} / \mathrm{Fe}$ et dans le cas du réacteur $\mathrm{Fe} / \mathrm{Fe}$, le $\mathrm{pH}$ du palier se situerait autour de 10 avec des fluctuations.

D'après la Figure 2, Le $\mathrm{pH}$ du rejet de teinture du tissu en coton se trouvant initialement à une valeur proche de 11 , diminue pendant les cinq premières minutes, puis augmente.

Evolution de la conductivité en fonction de temps pendant le traitement

Les Figures 5 et 6 présentent respectivement l'évolution de la conductivité en fonction du temps pendant la dépollution du rejet de teinture du tissu en polyester et du rejet de teinture du tissu en coton par électrocoagulation.

D'après la Figure 5, nous constatons pour l'ensemble des essais appliqués sur le rejet de polyester, la conductivité présente une légère augmentation, puis une stabilisation.

On observe, par ailleurs, que le réacteur $\mathrm{Fe} / \mathrm{Fe}$ présente une différence de conductivité par rapport aux deux autres réacteurs.

D'après la Figure 6, nous constatons une augmentation de la conductivité pour l'ensemble des essais appliqués sur le rejet de coton.

Rendement d'élimination en DCO en fonction du temps pendant le traitement

Les Figures 7 et 8 présentent respectivement le rendement d'élimination de la DCO en fonction du temps pendant la dépollution du rejet de teinture du tissu en polyester et du rejet de teinture du tissu en coton par électrocoagulation.

Les courbes de la Figure 7, sont constituées de trois parties, la première est ascendante, une deuxième est descendante et une troisième ayant l'allure palier, notamment pour les réacteurs de $\mathrm{Fe} / \mathrm{Al}$ et $\mathrm{Al} / \mathrm{Al}$, mais le réacteur à deux plaques de fer, présente une allure variable.

Lors des premières minutes, le rendement d'élimination atteint $60 \%$ pour le mélange, alors que pour l'aluminium seul, le rendement d'élimination atteint une valeur de $50 \%$, et pour le fer seul le rendement est de $30 \%$ seulement. Donc le mélange 
fer/aluminium nous permet d'obtenir un meilleur rendement en fonction du temps de traitement.

Le rendement d'élimination atteint une valeur de $70 \%$ pour l'aluminium seul au bout de 50 minutes, prés de $60 \%$ pour le mélange fer/aluminium et $45 \%$ pour le fer seul au bout de $30 \mathrm{~min}$.

D'après les courbes de la Figure 8, nous avons constaté que le rendement en DCO augmente vers une valeur de $30 \%$ pour l'aluminium seul, de $45 \%$ pour le mélange fer/aluminium et d'une valeur de $28 \%$ pour le fer seul. Ainsi, on remarque qu'au bout de 30 minutes le pourcentage d'élimination de la DCO diminue dans le cas de l'aluminium et fer/aluminium.

Rendement de la décoloration en fonction du temps pendant le traitement

Les Figures 9 et 10 présentent respectivement le rendement de la décoloration en fonction du temps pendant la dépollution du rejet de teinture du tissu en polyester et du rejet de teinture du tissu en coton par électrocoagulation.

D'après la Figure 9, nous avons constaté que le pourcentage de la décoloration augmente après un temps de 15 minutes.

D'après la Figure 10, nous avons constaté que le pourcentage de la décoloration augmente continuellement.

D'après cette même figure, nous avons remarqué que le réacteur qui constitue des électrodes de $\mathrm{Fe} / \mathrm{Al}$ présente une meilleure disposition pour le traitement de rejet de teinture du tissu en coton de $\mathrm{pH}$ basique.

Traitement du rejet par adsorption sur des composés synthétiques préparés par électrocoagulation

Evolution de pH en fonction de la concentration des composés synthétiques ajoutés.

Les Figures 11 et 12 présentent respectivement l'évolution du $\mathrm{pH}$ en fonction de la concentration des composés synthétiques (A3, AF2, F1) préparés par électrocoagulation de granulométrie $(160 \mu \mathrm{m}$ $<\mathrm{X}<500 \mu \mathrm{m}$ et de $\mathrm{X}<160 \mu \mathrm{m})$ pendant la dépollution du rejet de teinture du tissu en polyester.

D'après les Figures 11 et 12 , nous avons constaté dans le cas d'une granulométrie moyenne, le $\mathrm{pH}$ augmente pour atteindre une valeur de 7.5 après l'ajout d'une masse de coagulant correspondant à une concentration de $2,5 \mathrm{~g} / \mathrm{l}$, puis se stabilise vers un $\mathrm{pH}$ proche de 7 .

Dans le cas d'une granulométrie fine, le pH augmente jusqu' à une valeur de 6 , puis se stabilise en laissant prévoir une grande prédominance de l'hydroxyde après l'ajout d'une masse de coagulant correspondant à une concentration de 2,5 g/l.

Les Figures 13 et 14 présentent respectivement l'évolution du $\mathrm{pH}$ en fonction de la concentration des composés synthétiques (A3, AF2, F1) préparés par électrocoagulation de granulométrie $(160 \mu \mathrm{m}$ $<\mathrm{X}<500 \mu \mathrm{m}$ et de $\mathrm{X}<160 \mu \mathrm{m})$ pendant la dépollution du rejet de teinture du tissu en coton.

D'après les Figures 13 et 14 , nous avons remarqué que, pour l'ensemble des essais, le $\mathrm{pH}$ diminue lors de l'ajout du coagulant. Cette diminution augmente avec la dose de ce coagulant.

Le $\mathrm{pH}$ atteint une valeur proche de 7 pour l'aluminium seul et de 9.5 pour le fer seul et le mélange fer/aluminium.

Evolution de la conductivité en fonction de la concentration des composés synthétiques ajoutés

Les Figures 15 et 16 présentent respectivement l'évolution de la conductivité en fonction de la concentration des composés synthétiques (A3, AF2, F1) préparés par électrocoagulation de granulométrie $(160 \mu \mathrm{m}$ $<\mathrm{X}<500 \mu \mathrm{m}$ et de $\mathrm{X}<160 \mu \mathrm{m})$ pendant la dépollution du rejet de teinture du tissu en polyester.

Pour l'ensemble des essais de traitement du rejet de teinture du tissu en polyester (Figures 15 et 16), la conductivité 
augmente jusqu'à une valeur de $1,1 \mathrm{~ms} / \mathrm{cm}$ en fonction des masses ajoutées des composés synthétiques A3, F1 et AF2 dans les deux cas de granulométrie. Cette dernière semble plus prononcée pour les coagulants synthétiques à base de fer que celle à base d'aluminium.

Les Figures 17 et 18 présentent respectivement l'évolution de la conductivité en fonction de la concentration des composés synthétiques préparés par électrocoagulation de granulométrie $(160 \mu \mathrm{m}<\mathrm{X}<500 \mu \mathrm{m}$ et de $\mathrm{X}<160 \mu \mathrm{m})$ pendant la dépollution du rejet de teinture du tissu en coton.

Pour l'ensemble des essais de traitement du rejet de teinture du tissu en coton (Figures 17 et 18), la conductivité augmente jusqu'à une valeur de $1,4 \mathrm{~ms} / \mathrm{cm}$ en fonction des masses ajoutées des composés synthétiques A3, F1 et AF2 dans le cas d'une granulométrie faible.

On observe, en particulier, dans la Figure 18, que le composé à base d'aluminium, libère plus d'ions (conductivité élevée) que le coagulant à base de fer.

Rendement d'élimination en DCO en fonction de la concentration des composés synthétiques ajoutés

Les Figures 19 et 20 présentent respectivement l'évolution du rendement d'élimination en DCO en fonction de la concentration des composés synthétiques (A3, AF2, F1) préparés par électrocoagulation de granulométrie $(160 \mu \mathrm{m}<\mathrm{X}<500 \mu \mathrm{m}$ et de $\mathrm{X}<$ $160 \mu \mathrm{m}$ ) pendant la dépollution du rejet de teinture du tissu en polyester.

Dans le cas de traitement de rejet de polyester, on constate que le coagulant synthétique à base d'aluminium présente des capacités d'adsorption plus faibles que ceux à base du fer et de l'aluminium et du fer et ce pour les deux granulométries.

Pour la granulométrie moyenne (160 $\mu \mathrm{m}<\mathrm{X}<500 \mu \mathrm{m})$ (Figure 19), le rendement en DCO augmente et ne dépasse pas une valeur de $10 \%$ pour une concentration de $5 \mathrm{~g} / \mathrm{l}$ dans le cas de l'aluminium seul (A3). Par contre pour le cas du mélange fer/aluminium (AF2), le rendement en DCO atteint une valeur de $45 \%$ pour la même concentration de coagulant de même que le fer seul (F1) il donne un rendement de $42 \%$ à 2 g/l.

Le meilleur rendement est obtenu par le mélange fer/aluminium (AF2) (un rendement $45 \%$ en termes de DCO pour une concentration de $5 \mathrm{~g} / \mathrm{l})$.

Pour la granulométrie fine $(\mathrm{X}<160 \mu \mathrm{m})$ (Figure 20), le rendement en DCO ne dépasse pas les $10 \%$ pour une concentration du coagulant de $1 \mathrm{~g} / \mathrm{l}$ dans le cas de l'aluminium seul (A3), et est de seulement $11 \%$ pour une concentration de $5 \mathrm{~g} / \mathrm{l}$ du même coagulant.

Dans le cas du mélange fer/aluminium(AF2), le rendement en DCO atteint une valeur de $40 \%$ pour une concentration de $1 \mathrm{~g} / \mathrm{l}$ de coagulant, et augmente jusqu'à $50 \%$ pour une concentration de $5 \mathrm{~g} / \mathrm{l}$ du coagulant. Un rendement de $45 \%$ est obtenu pour une concentration de $1 \mathrm{~g} / \mathrm{l}$ en coagulant de Fer seul (F1).

Les Figures 21 et 22 présentent respectivement l'évolution du rendement d'élimination en DCO en fonction de la concentration des composés synthétiques (A3, AF2, F1) préparés par électrocoagulation de granulométrie $(160 \mu \mathrm{m}<\mathrm{X}<500 \mu \mathrm{m}$ et de $\mathrm{X}<$ $160 \mu \mathrm{m})$ pendant la dépollution du rejet de teinture du tissu en coton.

Pour l'ensemble des essais dans le cas du traitement du rejet de coton (Figures 21 et 22), le rendement en DCO augmente jusqu'à une valeur maximale de $50 \%$ pour une concentration de $40 \mathrm{~g} / \mathrm{l}$ pour les deux types de granulométrie. Les trois coagulants synthétiques donnent une élimination en DCO à peu près de même rapport.

Rendement de décoloration en fonction de la concentration des composés synthétiques ajoutés

Les Figures 23 et 24 présentent respectivement l'évolution du rendement de décoloration en fonction de la concentration des composés synthétiques (A3, AF2, F1) préparés par électrocoagulation de 
granulométrie $(160 \mu \mathrm{m}<\mathrm{X}<500 \mu \mathrm{m}$ et de $\mathrm{X}<$ $160 \mu \mathrm{m})$ pendant la dépollution du rejet de teinture du tissu en polyester.

Pour l'ensemble des essais de rejet du polyester (Figures 23 et 24), le pourcentage de décoloration augmente jusqu'à une valeur de $33 \%$, après l'ajout d'une masse de coagulant à base d'aluminium seul, correspondant à une concentration de 2,5 g/l pour la granulométrie moyenne et 1,2 $\mathrm{g} / \mathrm{l}$ pour la granulométrie fine, puis se stabilise à cette valeur lorsque l'adsorption se fait sur les hydroxydes formés.

Les Figures 25 et 26 présentent respectivement l'évolution du rendement de décoloration en fonction de la concentration des composés synthétiques (A3, AF2, F1) préparés par électrocoagulation de granulométrie $(160 \mu \mathrm{m}<\mathrm{X}<500 \mu \mathrm{m}$ et de $\mathrm{X}<$ $160 \mu \mathrm{m})$ pendant la dépollution du rejet de teinture du tissu en coton.
Pour l'ensemble des essais de rejet du coton (Figures 25 et 26), nous avons constaté que: Dans le cas de la granulométrie moyenne $(160 \mu \mathrm{m}<\mathrm{X}<500 \mu \mathrm{m})$ (Figure 25), le pourcentage de décoloration augmente jusqu'à une valeur maximale de $40 \%$ pour $\mathrm{F} 1$, $\mathrm{AF} 2$ et $\mathrm{A} 3$ après l'ajout d'une concentration de $10 \mathrm{~g} / \mathrm{l}$ pour $\mathrm{F} 1$ et $\mathrm{AF} 2$ et seulement d'une concentration de $5 \mathrm{~g} / \mathrm{l}$ pour A3.

Dans le cas de la granulométrie faible $(\mathrm{X}<160 \mu \mathrm{m})$ (Figure 26), le pourcentage de décoloration augmente jusqu'à une valeur de $40 \%$ pour $\mathrm{F} 1,48 \%$ pour $\mathrm{AF} 2$ et $60 \%$ pour $\mathrm{A} 3$ après l'ajout d'une concentration de $5 \mathrm{~g} / \mathrm{l}$, après une stabilisation vers cette valeur.

D'autre côté, l'aluminium montre une bonne efficacité d'élimination du colorant (70\% pour $20 \mathrm{~g} / \mathrm{l})$.

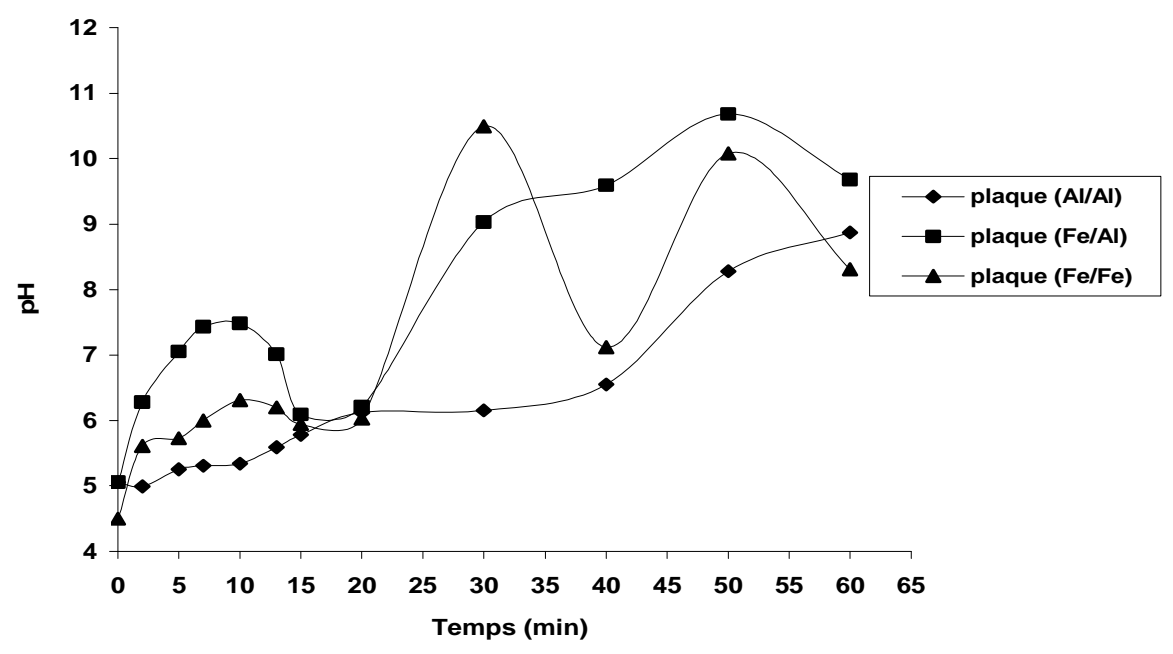

Figure 1: Evolution de $\mathrm{pH}$ en fonction du temps pendant la dépollution du rejet de teinture du tissu en polyester par électrocoagulation avec :

-Plaque (Al/Al) : Réacteur avec deux plaques d'aluminium.

-Plaque (Fe/Al) : Réacteur avec une plaque de fer et une d'aluminium.

-Plaque (Al/Al) : Réacteur avec deux plaques de fer. 


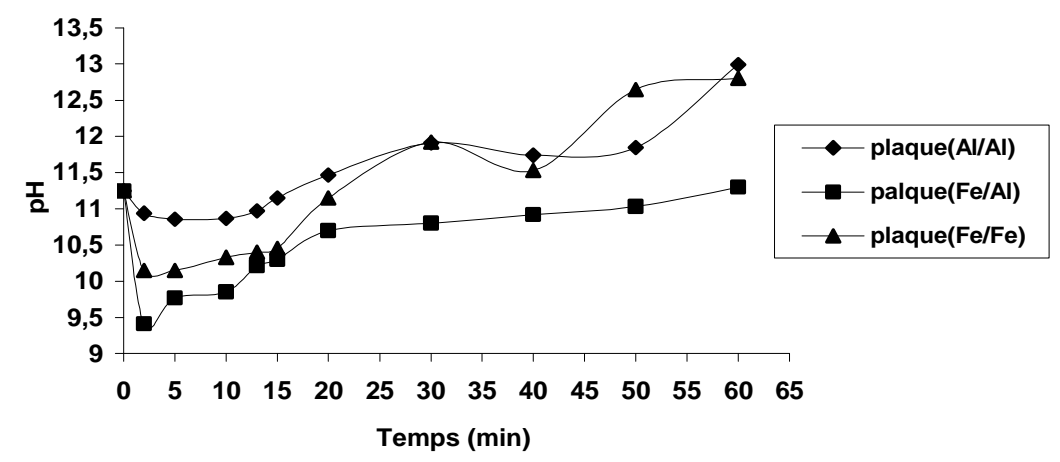

Figure 2: Evolution de $\mathrm{pH}$ en fonction du temps pendant la dépollution du rejet de teinture du tissu en coton par électrocoagulation.

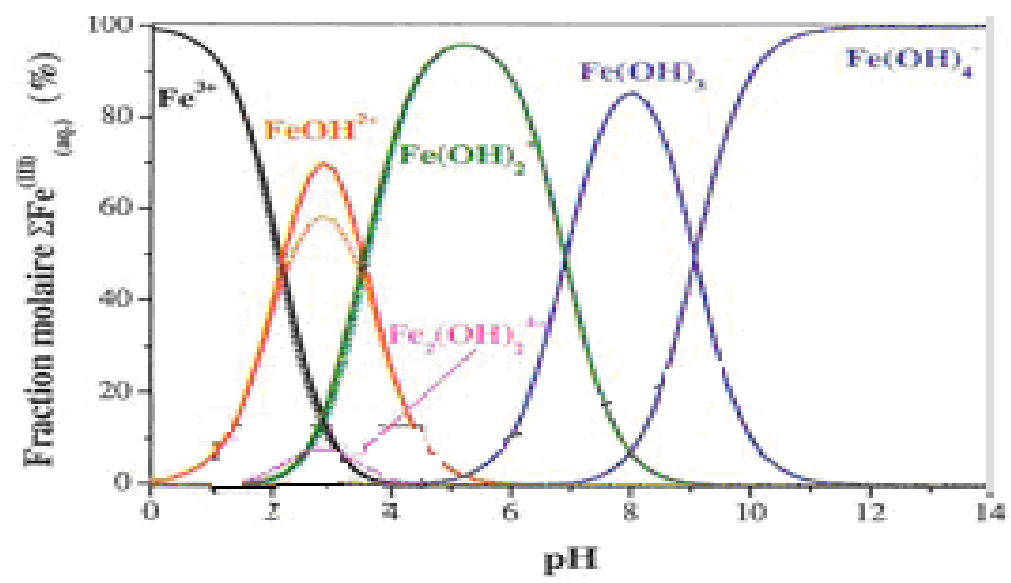

Figure 3: Répartition molaire du fer (III) en fonction du pH en eau pure et en conditions standards.

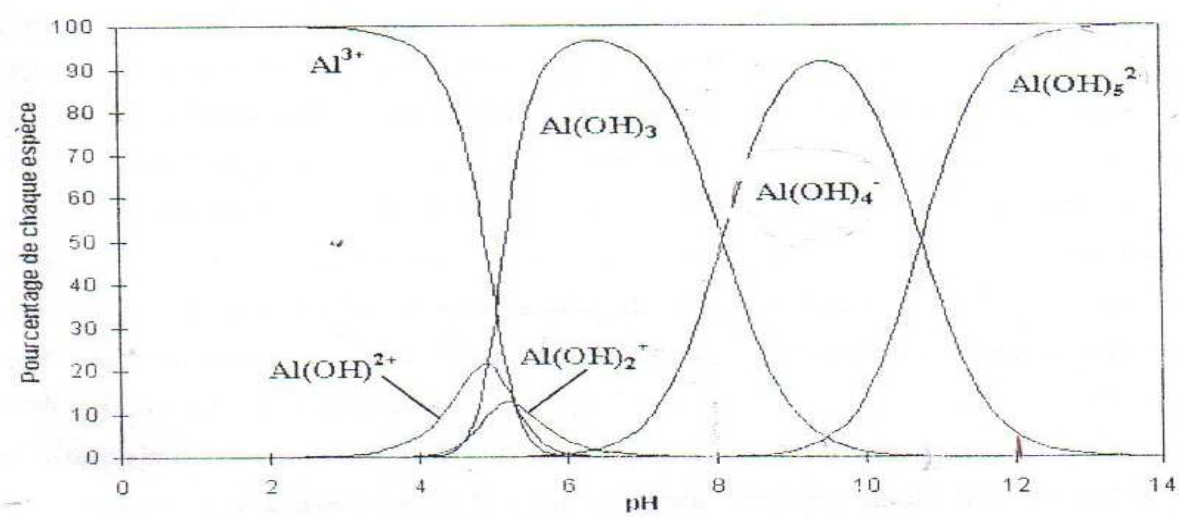

Figure 4 : Répartition des espèces hydrolysées de l'aluminium en fonction du pH. 


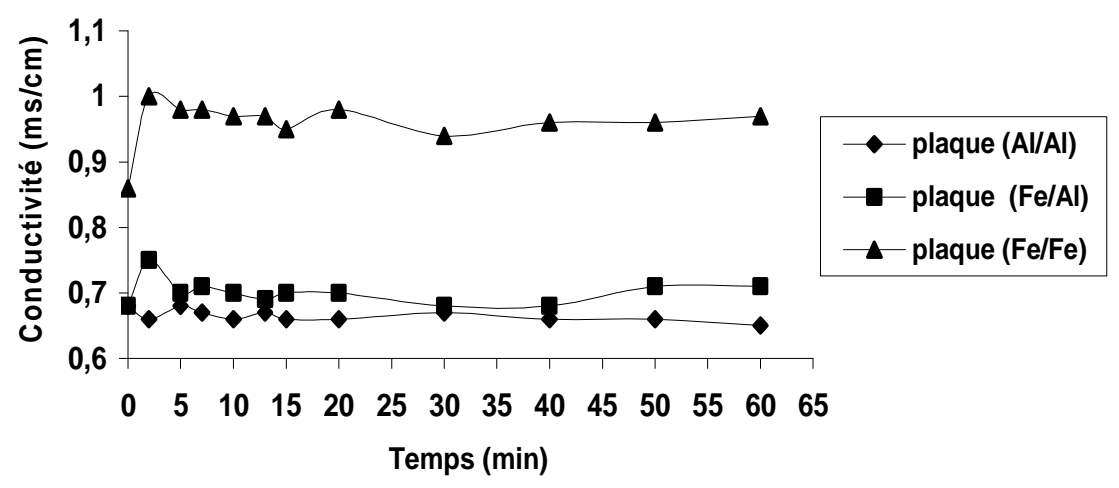

Figure 5 : Evolution de la conductivité en fonction du temps pendant la dépollution du rejet de teinture du tissu en polyester par électrocoagulation.

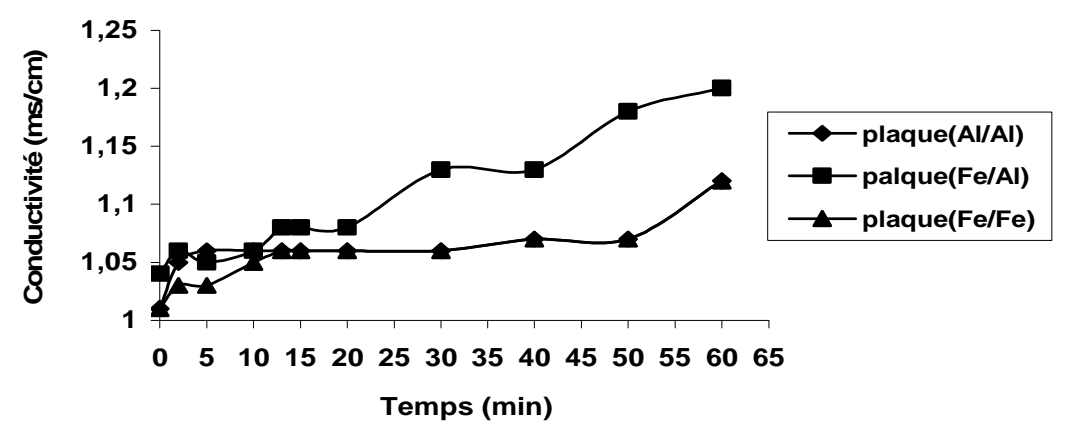

Figure 6 : Evolution de la conductivité en fonction du temps pendant la dépollution du rejet de teinture du tissu en coton par électrocoagulation.

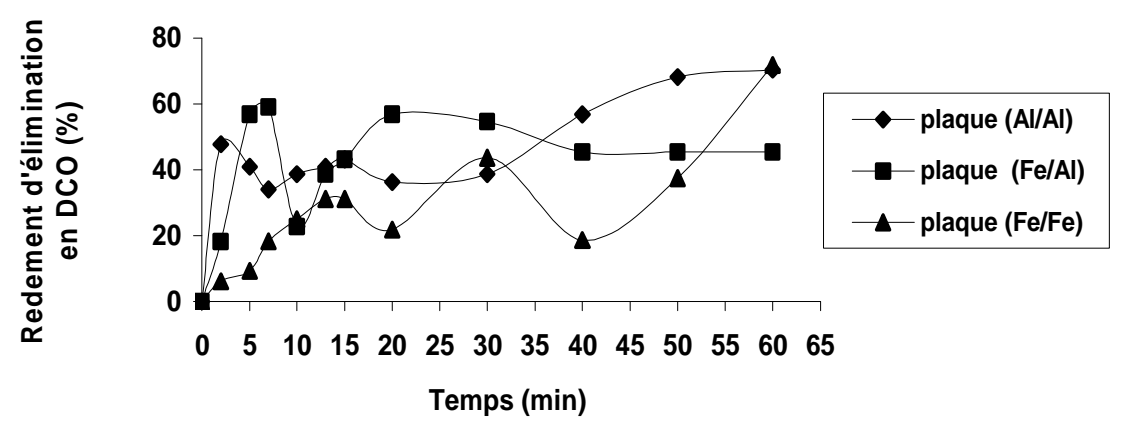

Figure 7 : Rendement d'élimination en DCO en fonction du temps pendant la dépollution du rejet de teinture du tissu en polyester par électrocoagulation. 

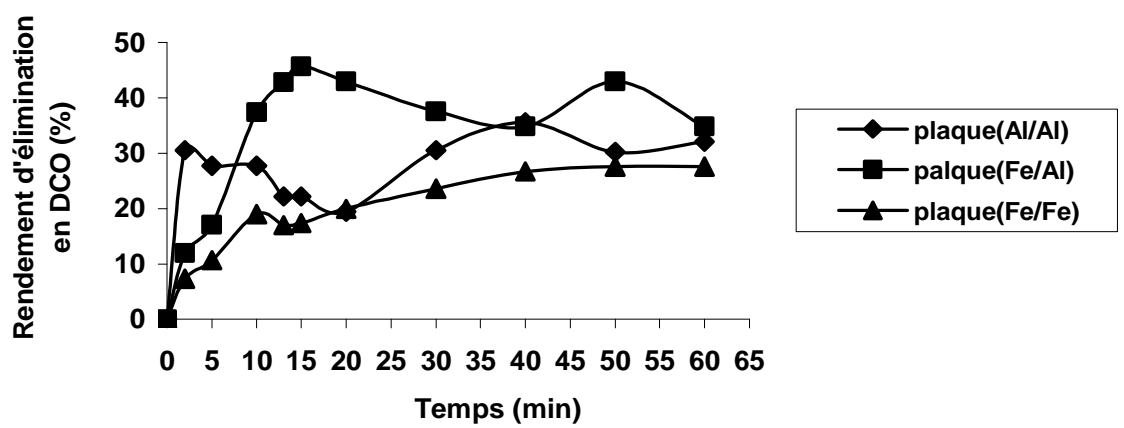

Figure 8 : Rendement d'élimination en DCO en fonction du temps pendant la dépollution du rejet de teinture du tissu en coton par électrocoagulation.

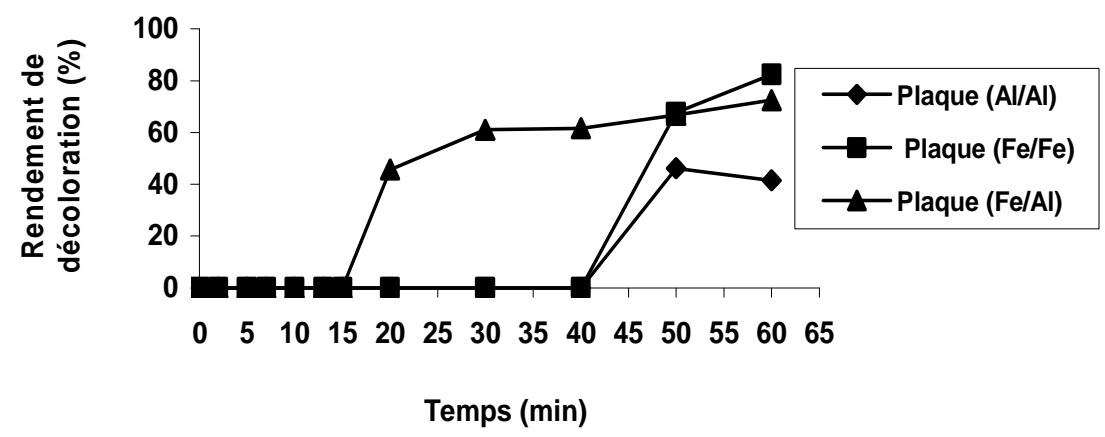

Figure 9 : Rendement de décoloration en fonction de temps pendant la dépollution du rejet de teinture du tissu en polyester par électrocoagulation.

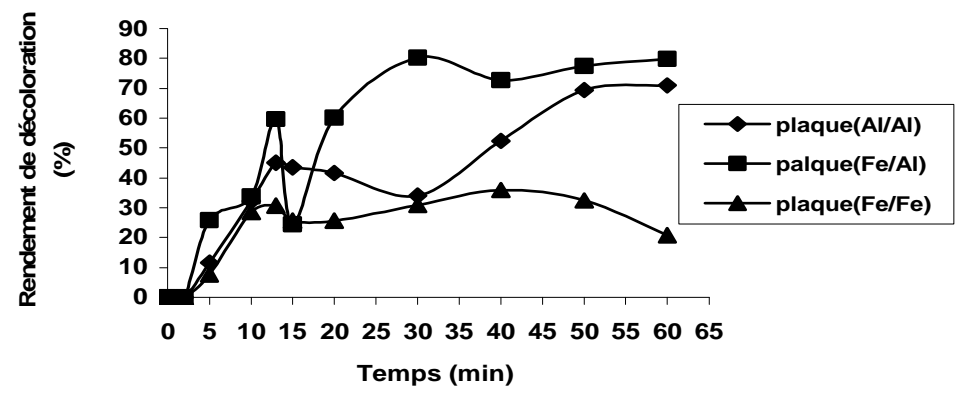

Figure 10 : Rendement de décoloration en fonction de temps pendant la dépollution du rejet de teinture du tissu en coton par électrocoagulation. 


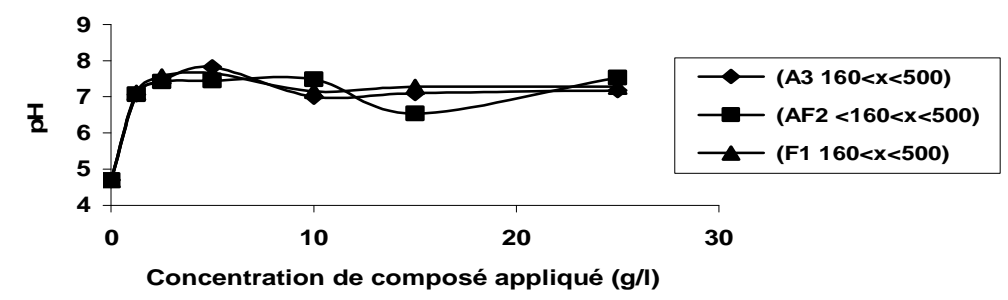

Figure 11 : Evolution de $\mathrm{pH}$ en fonction de la concentration des composés synthétiques préparés par électrocoagulation de granulométrie $(160 \mu \mathrm{m}<\mathrm{X} 500 \mu \mathrm{m})$ pendant la dépollution du rejet de teinture du tissu en polyester par adsorptions sur ces composés avec :

- A3: composé à base d'aluminium seul, préparé en utilisant quatre plaques d'aluminium.

- F1 : composé à base de fer seul, préparé en utilisant quatre plaques de fer.

- AF2 : composé à base de mélange fer/aluminium, préparé en utilisant deux plaques de fer et deux plaques d'Aluminium.

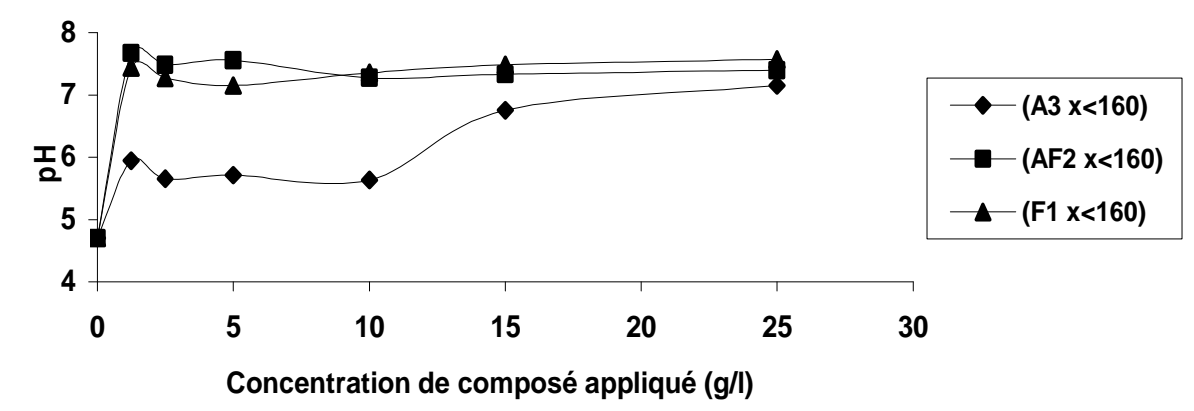

Figure 12: Evolution de $\mathrm{pH}$ en fonction de la concentration des composés synthétiques préparés par électrocoagulation de granulométrie $(\mathrm{X}<160 \mu \mathrm{m})$ pendant la dépollution du rejet de teinture $\mathrm{du}$ tissu en polyester par adsorptions sur ces composés.

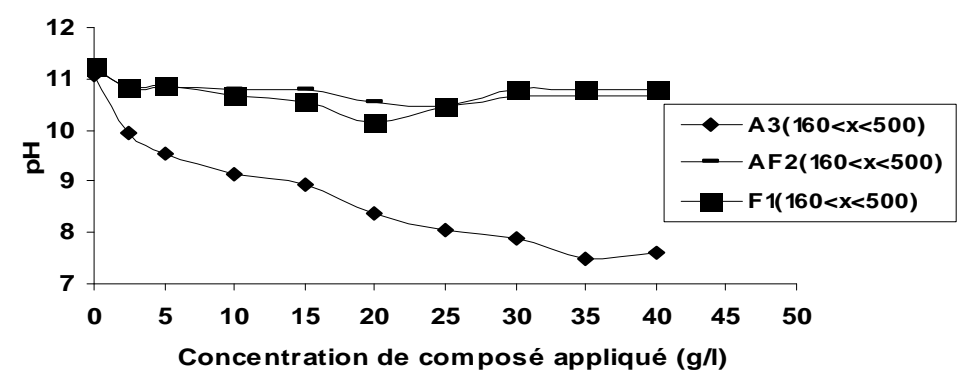

Figure 13 : Evolution de $\mathrm{pH}$ en fonction de la concentration des composés synthétiques préparés par électrocoagulation de granulométrie $(160 \mu \mathrm{m}<\mathrm{X}<500 \mu \mathrm{m})$ pendant la dépollution du rejet de teinture du tissu en coton par adsorptions sur ces composés. 


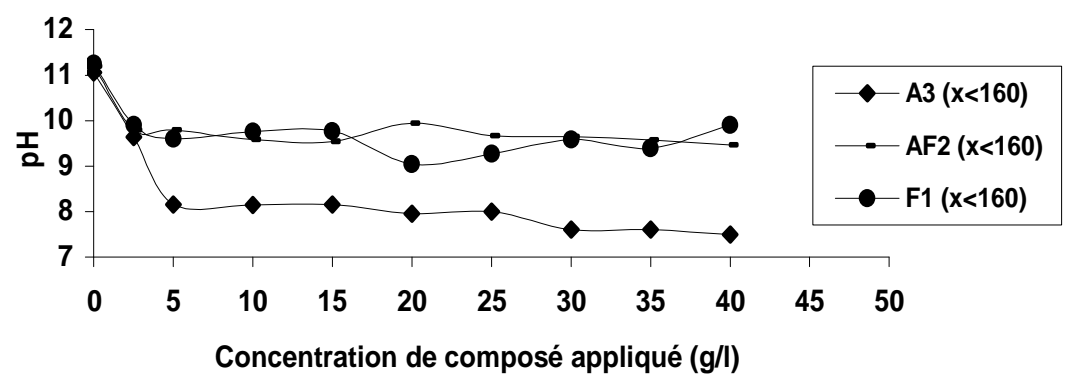

Figure 14 : Evolution de $\mathrm{pH}$ en fonction de la concentration des composés synthétiques préparés par électrocoagulation de granulométrie $(X<160 \mu \mathrm{m})$ pendant la dépollution du rejet de teinture du tissu en coton par adsorptions sur ces composés.

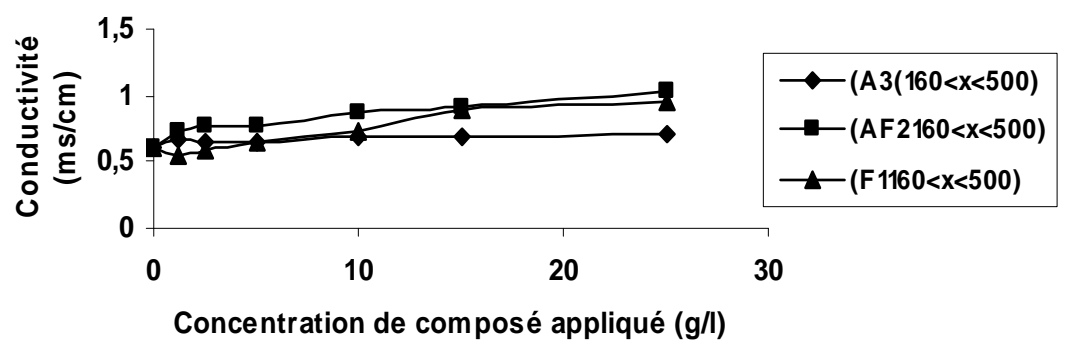

Figure 15 : Evolution de la conductivité en fonction de la concentration des composés synthétiques préparés par électrocoagulation de granulométrie $(160 \mu \mathrm{m}<\mathrm{X}<500 \mu \mathrm{m})$ pendant la dépollution $\mathrm{du}$ rejet de teinture du tissu en polyester par adsorptions sur ces composés.

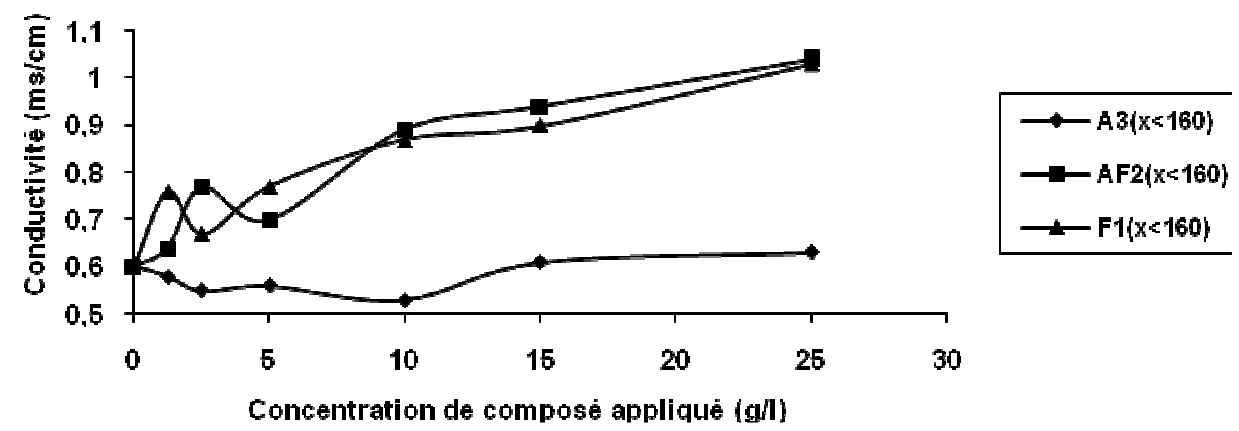

Figure 16 : Evolution de la conductivité en fonction de la concentration des composés synthétiques préparés par électrocoagulation de granulométrie $(X<160 \mu \mathrm{m})$ pendant la dépollution du rejet de teinture du tissu en polyester par adsorptions sur ces composés. 


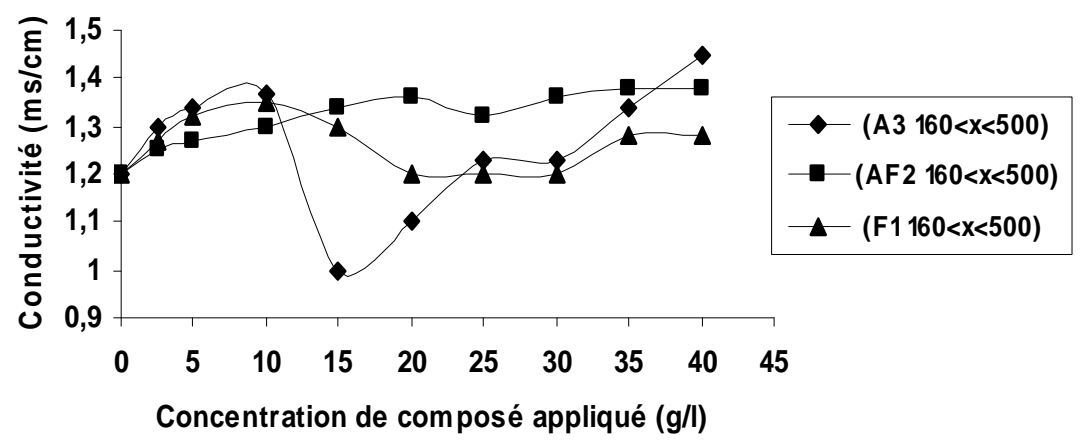

Figure 17 : Evolution de la conductivité en fonction de la concentration des composés synthétiques préparés par électrocoagulation de granulométrie $(160 \mu \mathrm{m}<\mathrm{X}<500 \mu \mathrm{m})$ pendant la dépollution $\mathrm{du}$ rejet de teinture du tissu en coton par adsorptions sur ces composés.

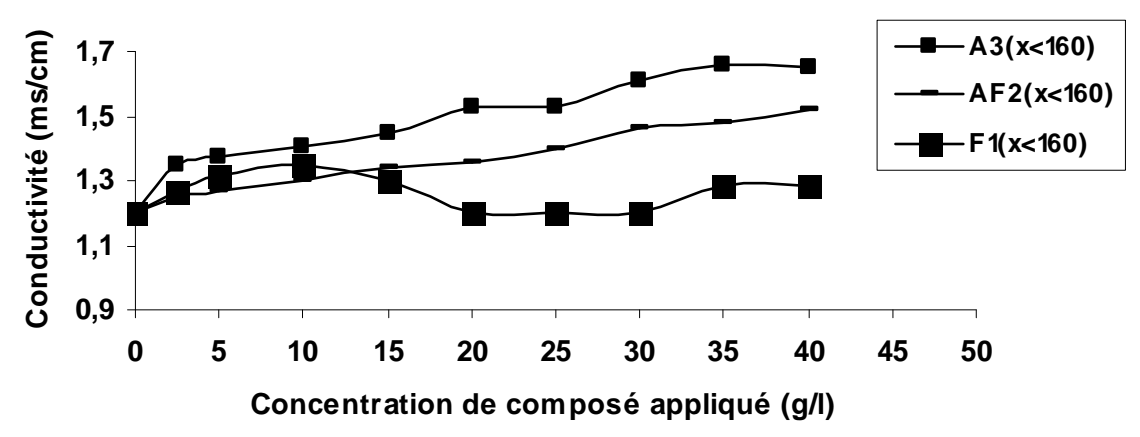

Figure 18: Evolution de la conductivité en fonction des concentrations ajoutées des composés synthétiques $(\mathrm{A} 3, \mathrm{AF} 2, \mathrm{~F} 1)$ de granulométrie $(\mathrm{X}<160 \mu \mathrm{m})$ pendant la dépollution du rejet de teinture du tissu en coton par adsorptions sur ces composés.

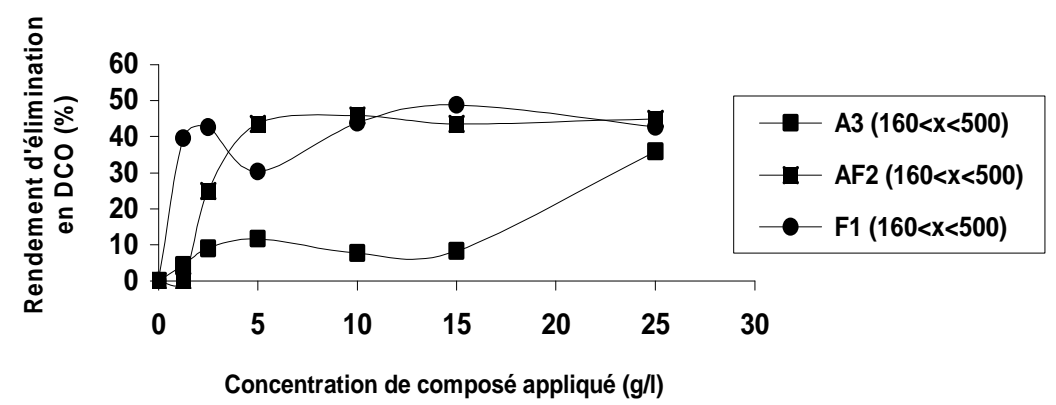

Figure 19: Rendement d'élimination en DCO en fonction des concentrations ajoutées des composés synthétiques (A3, AF2, F1) de granulométrie $(160 \mu \mathrm{m}<\mathrm{X}<500 \mu \mathrm{m})$ pendant la dépollution du rejet de teinture du tissu en polyester par adsorptions sur ces composés. 


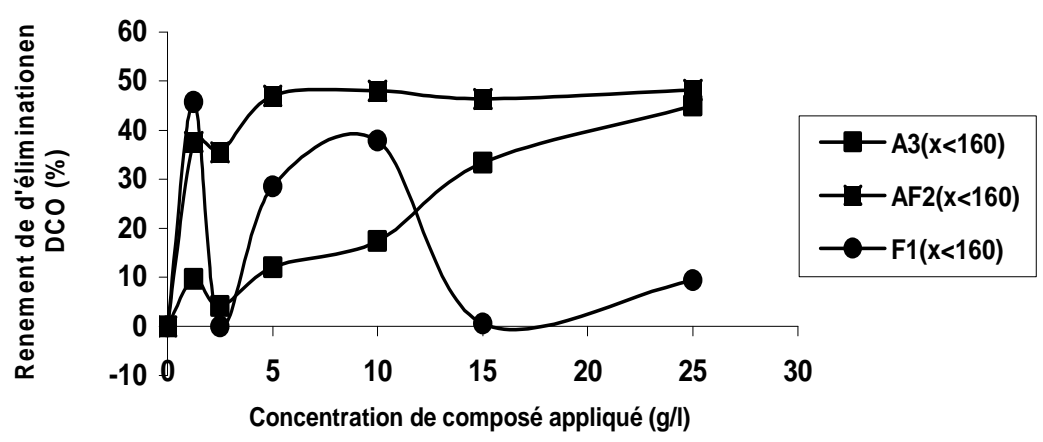

Figure 20 : Rendement d'élimination en DCO en fonction de la concentration des composés synthétiques $(\mathrm{A} 3, \mathrm{AF} 2, \mathrm{~F} 1)$ de granulométrie $(\mathrm{X}<160 \mu \mathrm{m})$ pendant la dépollution du rejet de teinture du tissu en polyester par adsorptions sur ces composés.

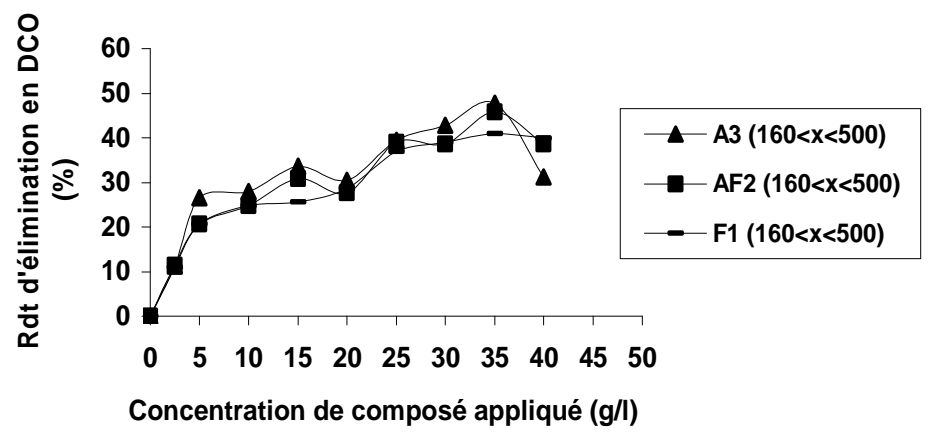

Figure 21: Evolution d'élimination en DCO en fonction de la concentration des composés synthétiques des composés synthétiques (A3, AF2, F1) de granulométrie $(160 \mu \mathrm{m}<\mathrm{X}<500 \mu \mathrm{m})$ pendant la dépollution du rejet de teinture du tissu en coton par adsorptions sur ces composés.

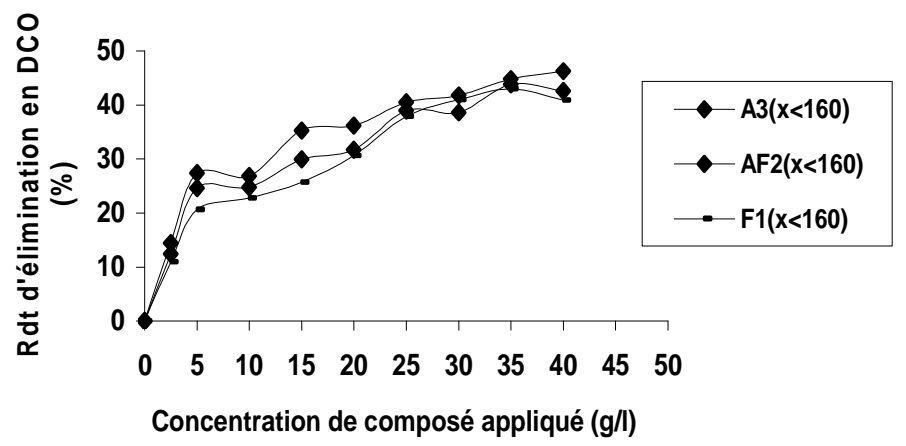

Figure 22: Evolution d'élimination en DCO en fonction de la concentration en fonction de la concentration des composés synthétiques $(\mathrm{A} 3, \mathrm{AF} 2, \mathrm{~F} 1)$ de granulométrie $(\mathrm{X}<160 \mu \mathrm{m})$ pendant la dépollution du rejet de teinture du tissu en coton par adsorptions sur ces composés. 


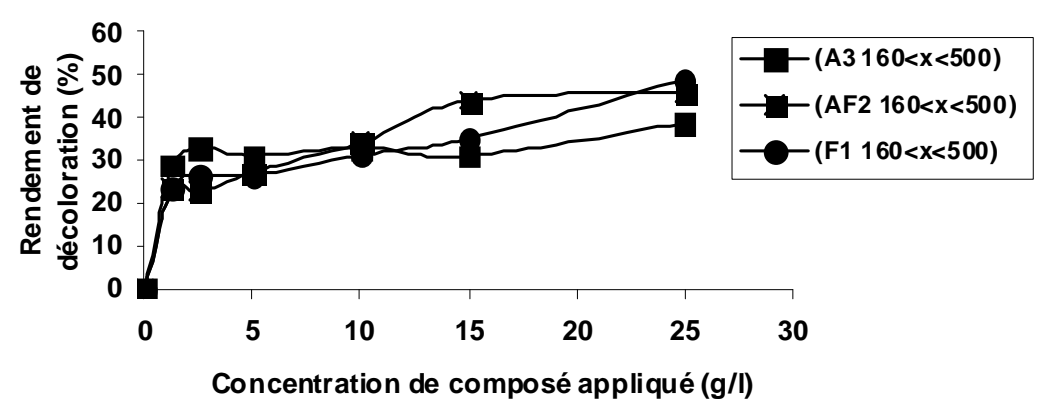

Figure 23 : Rendement de décoloration en fonction de la concentration des composés synthétiques préparés par électrocoagulation de granulométrie $(160 \mu \mathrm{m}<\mathrm{X}<500 \mu \mathrm{m})$ pendant la dépollution $\mathrm{du}$ rejet de teinture du tissu en polyester par adsorptions sur ces composés.

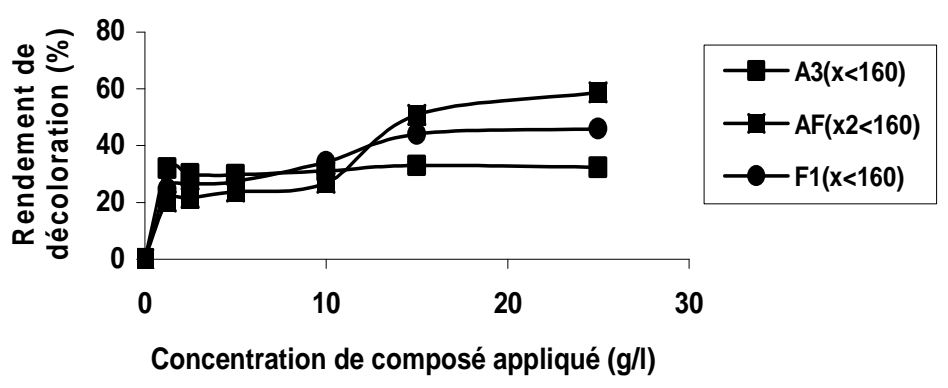

Figure 24 : Rendement de décoloration en fonction de la concentration des composés synthétiques de granulométrie $(X<160 \mu \mathrm{m})$ pendant la dépollution du rejet de teinture du tissu en polyester par adsorptions sur ces composés.

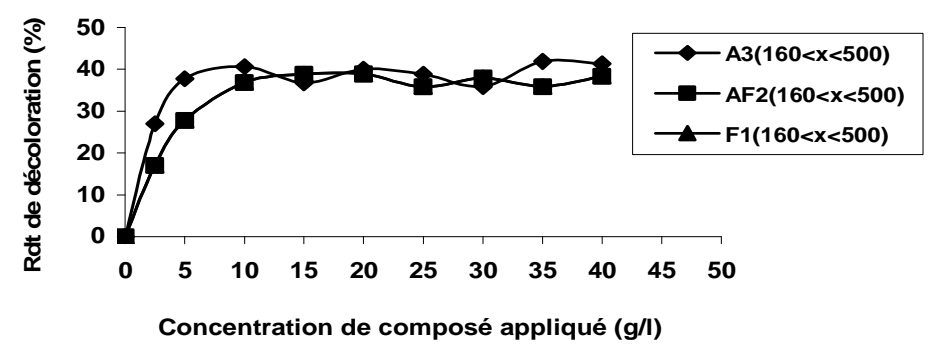

Figure 25 : Rendement de décoloration en fonction de la concentration des composés synthétiques préparés par électrocoagulation de granulométrie $(160 \mu \mathrm{m}<\mathrm{X}<500 \mu \mathrm{m})$ pendant la dépollution du rejet de teinture du tissu en coton par adsorptions sur ces composés. 


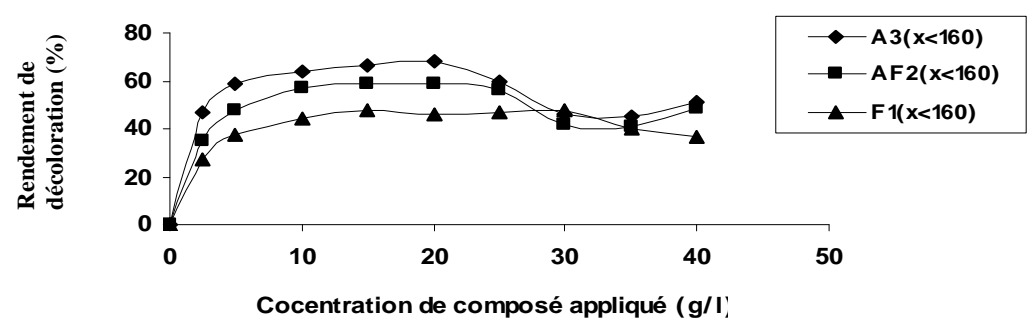

Figure 26 : Rendement de décoloration en fonction de la concentration des composés synthétiques préparés par électrocoagulation de granulométrie $(\mathrm{X}<160 \mu \mathrm{m})$ pendant la dépollution du rejet de teinture du tissu en coton par adsorptions sur ces composés.

\section{DISCUSSION}

Traitement du rejet par électrocoagulation Evolution du pH en fonction du temps pendant le traitement

Dans le cas du rejet en polyester (Figure 1), à pH acide d'une valeur de 4,7, l'augmentation pendant les dix premières minutes, peut être expliquée par la dissolution des électrodes de fer et/ou d'aluminium à l'anode pour former des espèces monomères ou polymères selon la réaction (I) (Parks, 1972):

$\mathrm{xMe}^{3+}+\mathrm{yH}_{2} \mathrm{O} \rightleftarrows \mathrm{Me}_{\mathrm{x}}(\mathrm{OH})^{(3 \mathrm{x}-\mathrm{y})+}+\mathrm{yH}^{+}$

avec Me: Al, Fe ...................; ( I )

La disponibilité des ions ${ }^{-} \mathrm{OH}$ résultant de la complexation des cations métalliques par les molécules organiques disponibles dans le milieu (Réaction II) (Ben Tahar et al., 2004), ce qui entraîne l'augmentation de $\mathrm{pH}$.

$\mathrm{Me}^{3+}+\mathrm{n} \mathrm{M}_{\text {Org }} \longleftarrow\left[\mathrm{Me}\left(\mathrm{M}_{\mathrm{Org}}\right) \mathrm{n}\right]^{3+} \ldots$...(II) Avec $\mathrm{M}_{\text {org }}$ : matière organique; $\mathrm{Me}^{3+}$ : métal de fer ou d'aluminium.

Au cours du temps, et lorsque la concentration des ions hydroxyles augmente, les molécules de colorant sont remplacées par les ions ${ }^{-} \mathrm{OH}$ ce qui diminue la valeur $\mathrm{du} \mathrm{pH}$ (réaction III) (Torrades et al., 2004) :

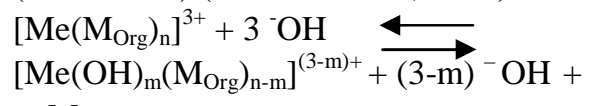
$\mathrm{m} \mathrm{M}_{\mathrm{Org}}$

Le réacteur $\mathrm{Fe} / \mathrm{Al}$ présente une allure en cloche plus prononcée que celle du réacteur $\mathrm{Fe} / \mathrm{Fe}$, qui provient probablement du fait que la différence du potentiel normal entre les deux électrodes de fer et d'aluminium est en faveur de l'aluminium. Celui-ci s'ajoute au potentiel imposé au réacteur. Au bout de 20 minutes on atteint un palier de $\mathrm{pH}$ proche de 6 dans le cas de l'aluminium seul (zone de prédominance de $\mathrm{Al}(\mathrm{OH})_{3}$ (Figure 3) (Rebhum et Lurie, 1993), et de 9 dans le cas du mélange $\mathrm{Al} / \mathrm{Fe}$ (zone de prédominance de $\mathrm{Fe}(\mathrm{OH})_{3}$ ). Dans le cas du réacteur $\mathrm{Fe} / \mathrm{Fe}$, le $\mathrm{pH}$ du palier se situerait autour de 10 avec des fluctuations, qui pourraient être dues à la formation d'autres formes d'hydroxydes de fer (Figure 4) (Neff, 2003). Ces formes solides pourraient entraîner l'adsorption du polluant.

Dans le cas du rejet en coton (Figure2), à $\mathrm{pH}$ basique d'une valeur de $11, \mathrm{le} \mathrm{pH}$ initial diminue pendant les cinq premières minutes, puis augmente. Ceci peut s'expliquer par les deux réactions (Equations IV et V) (Bensaid, 2009).

En milieu basique les métaux peuvent se dissoudre, en donnant des aluminates ou ferrates et libérant de l'hydrogène qui va aider à l'élimination du polluant par électroflotation:
$2 \mathrm{Me}+6 \mathrm{H}_{2} \mathrm{O}+2 \mathrm{OH}^{-} \leftrightharpoons 2 \mathrm{Me}(\mathrm{OH})_{4}^{-}$ $+3 \mathrm{H}_{2}$ (IV)

Ce qui entraîne une diminution de $\mathrm{pH}$, jusqu'à des valeurs proches de 11 où des germes d'hydroxydes peuvent apparaître.

En milieu encore basique et en présence d'ions aluminates ou ferrates en excès, la réaction, ci-dessous, a lieu: 


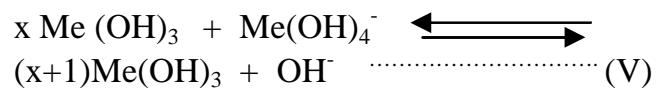

Montrant une libération d'ions ${ }^{-} \mathrm{OH}$, une augmentation de $\mathrm{pH}$ et la formation d'hydroxydes sur lesquels s'adsorbe le polluant et correspondant à un palier de $\mathrm{pH}$.

Evolution de la conductivité en fonction de temps pendant le traitement

Dans le cas du rejet en polyester (Figure 5), la légère augmentation de la conductivité est due probablement à l'enrichissement du milieu en ions cationiques (formes monomères et hydrolysées). La stabilité de la conductivité résulte d'un ensemble d'entités pouvant apparaître au cours du temps comme cela était démontré lors de l'interprétation de l'évolution du $\mathrm{pH}$ (Zidane et al., 2008).

Par ailleurs, le réacteur $\mathrm{Fe} / \mathrm{Fe}$ présente une différence de conductivité par rapport aux deux autres. Ceci pourrait s'expliquer par le potentiel normal du couple $\mathrm{Fe}^{2+} / \mathrm{Fe}$ permettant au fer de s'oxyder plus facilement en milieu aqueux.

Dans le cas du rejet en coton (Figure 6), une augmentation de la conductivité a lieu $\mathrm{du}$ fait de la présence des ions aluminium et fer, sous différentes formes, dissoutes continuellement de l'anode.

Rendement d'élimination en DCO en fonction du temps pendant le traitement

Dans le cas du rejet en polyester (Figure 7), les courbes sont constituées de trois parties, la première est ascendante, et peut être attribuée à l'élimination de la matière organique par coagulation électrostatique grâce aux espèces monomères ou hydrolysées (réaction I et II), une deuxième partie descendante, correspondant à la réaction d'échange III où la matière organique qui complexait le cation métallique, est rejetée en solution. Une troisième partie ayant l'allure palier, notamment pour les réacteurs de $\mathrm{Fe} / \mathrm{Al}$ et $\mathrm{Al} / \mathrm{Al}$, est probablement due à l'adsorption des colorants sur les hydroxydes qui se forment lors de l'échange de la matière organique par les ions hydroxyles (réaction III) sauf dans le cas du réacteur $\mathrm{Fe} / \mathrm{Fe}$ qui présente une allure variable due vraisemblablement à l'existence de plusieurs hydroxydes de fer.

Lors des premières minutes, le rendement d'élimination atteint $60 \%$ pour le mélange, alors que pour l'aluminium seul, le rendement d'élimination atteint une valeur de $50 \%$, et pour le fer seul le rendement est de $30 \%$ seulement. Donc le mélange fer/aluminium nous permet d'obtenir un meilleur rendement en fonction du temps de traitement.

Lorsque les réactions d'échanges ont lieu, l'adsorption se fait sur les hydroxydes de fer et d'aluminium et probablement d'autres structures solides formées (réaction III) (Fessinger, 1976).

Dans le cas du rejet en coton (Figure 8), l'augmentation du rendement en DCO pour les trois types de réacteurs peut s'expliquer par électro-flottation due au dégagement d'hydrogène à la cathode, permettant ainsi l'élimination des matières en suspension (MES).

Pour une valeur de $\mathrm{pH}$ donnée, $\mathrm{Me}(\mathrm{OH})_{3}$ qui se forme permet la diminution du potentiel $\xi$ et favorise alors l'élimination d'une partie de la DCO par adsorption.

$\mathrm{Au}$ bout de 30 minutes, la diminution du pourcentage de la DCO diminue dans le cas de l'aluminium et fer/aluminium peut s'expliquer par la stabilisation de la solution du fait de l'ennoiement des hydroxydes et par inversion de charge.

Rendement de la décoloration en fonction du temps pendant le traitement

Dans le cas du rejet en polyester (Figure 9), l'augmentation du pourcentage de la décoloration peut être expliqué par la disponibilité du $\mathrm{Me}(\mathrm{OH})_{3}$ qui se forme, favorisant ainsi une forte adsorption des colorants sur les hydroxydes. Ceci est conforme aux constatations faites lors de l'analyse du $\mathrm{pH}$ à travers lesquelles on a montré la présence de deux phases, la première où le $\mathrm{pH}$ augmente puis diminue, correspondant à la coagulation électrostatique et ensuite à l'élimination des matières organiques et la seconde où le $\mathrm{pH}$ augmente 
régulièrement favorisant la formation $\mathrm{du}$ précipité d'hydroxyde (Bensaid, 2009).

Dans le cas du rejet en coton (Figure 10), nous avons constaté que le pourcentage de la décoloration augmente continuellement. Ceci peut être expliqué par la disponibilité du $\mathrm{Me}(\mathrm{OH})_{3}$ qui se forme, favorisant ainsi une forte adsorption des colorants sur les hydroxydes.

Traitement du rejet par adsorption sur des composés synthétiques préparés par électrocoagulation

Evolution de pH en fonction de la concentration des composés synthétiques ajoutés.

Dans le cas des composés synthétiques d'une granulométrie moyenne utilisés pour le traitement de rejet de polyester, l'augmentation du $\mathrm{pH}$ après l'ajout d'une concentration de $2,5 \mathrm{~g} / \mathrm{l}$ puis une stabilisation vers un $\mathrm{pH}$ proche de 7 , peut être expliquée par la stabilité et la forte capacité d'adsorption des coagulants dans ce domaine de $\mathrm{pH}$. En effet les composés A3 (à base d'aluminium), AF2 (à base de fer/aluminium) et $\mathrm{F} 1$ (à base fer) sont principalement sous forme de $\mathrm{Me}(\mathrm{OH})_{3}$ (Zidane et al., 2008), qui présente un caractère amphotère et donne lieu à la réaction suivante (Equation $\mathrm{VI})$ :

$\mathrm{Me}(\mathrm{OH})_{3} \rightleftarrows \mathrm{Me}^{3+}+3 \mathrm{OH}^{-}$

D'après cette réaction on constate que les ions ${ }^{-} \mathrm{OH}$ sont libérés dans la solution par une partie de la matrice solide, qui se dissout partiellement. Les ions $\mathrm{Me}^{3+}$ sont alors complexés par les matières polluantes laissant les ions hydroxyles libres, ce qui explique l'augmentation du $\mathrm{pH}$ de 4,7 à un palier de 7, zone de prédominance de $\mathrm{Me}(\mathrm{OH})_{3}$, sur lequel s'adsorbe le polluant .

Dans le cas d'une granulométrie fine, l'augmentation du $\mathrm{pH}$ puis une stabilisation en laissant prévoir une grande prédominance de l'hydroxyde après l'ajout d'une masse de coagulant correspondant à une concentration de 2,5 g/l, peut être expliqué par la stabilisation des composés dans ce domaine de $\mathrm{pH}$, sur lesquels peut s'adsorber le polluant.
La granulométrie ne semble pas avoir d'impact dans cette zone du $\mathrm{pH}$ puisque la capacité d'adsorption est très élevée pour les deux types de granulométries.

Dans le cas des composés synthétiques d'une faible et moyenne granulométrie utilisés pour le traitement de rejet de coton (Figures 13 et 14), le $\mathrm{pH}$ diminue lors de l'ajout du coagulant vers une valeur proche de 7 pour l'aluminium seul (zone de prédominance de l'hydroxyde de $\left.\mathrm{Al}(\mathrm{OH})_{3}\right)$, et de 9.5 pour le fer seul et pour le mélange fer/aluminium (prédominance de l'hydroxyde de $\mathrm{Fe}(\mathrm{OH})_{3}$ ).

Le coagulant étant essentiellement constitué d'hydroxydes, celui -ci peut passer sous forme d'aluminates et de ferrates en consommant des ions hydroxyles (réaction IV) (Bensaid, 2008), et diminuant le $\mathrm{pH}$ jusqu'à une zone ou l'hydroxyde devient majoritaire et entraînant l'adsorption du polluant.

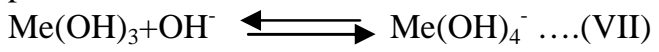

Cette diminution est peut être due à la réaction du métal en solution qui joue le rôle d'amphotère plus prononcé pour l'aluminium que pour le fer (réaction IV)

La granulométrie ne semble pas avoir d'impact dans ce domaine de $\mathrm{pH}$ ou la capacité d'adsorption est très élevée aussi bien pour la granulométrie fine que moyenne.

Evolution de la conductivité en fonction de la concentration des composés synthétiques ajoutés

L'augmentation de la conductivité pour l'ensemble des essais de traitement de rejet de teinture du tissu en polyester (Figures 15 et 16), peut être expliquée par la dissolution partielles des composés en libérant des ions $\mathrm{Al}^{3+}, \mathrm{Fe}^{3+}$ et ${ }^{-} \mathrm{OH}$.

L'augmentation de la conductivité pour l'ensemble des essais de traitement de rejet de teinture du tissu en polyester (Figures 17et 18), peut être expliquée par la formation du ferrate et aluminate dans le milieu basique.

Dans la Figure 18, le composé à base d'aluminium, libère plus d'ions (conductivité élevée) que le coagulant à base de fer. Ceci peut être dû à la facilité de consommation des ions hydroxyles et à la formation d'ions 
aluminates ou ferrates dans un rejet ayant un pH très basique (variété allotropiques, stabilité cristalline, caractère amphotère, ...). Cette constatation pourrait corroborer les résultats concernant le $\mathrm{pH}$.

Rendement d'élimination en DCO en fonction de la concentration des composés synthétiques ajoutés

Dans le cas du traitement de rejet de polyester, le coagulant synthétique à base d'aluminium présente des capacités d'adsorption plus faibles que ceux à base du fer/aluminium et du fer pour les deux types de granulométrie.

Ces valeurs de rendements variables peuvent être expliquées par une compétition entre les matières colorantes et organiques sur les sites d'adsorption existants dans les coagulants dont les hydroxydes métalliques peuvent présenter de nombreuses variétés allotropiques.

Il semble que la granulométrie n'a pas d'effet particulier sur la quantité de polluant adsorbé dans ce domaine de $\mathrm{pH}$, contrairement à ce qu'on attendait d'un tel essai. Il semblerait qu'il y ait d'autres mécanismes qui s'ajouteraient à l'adsorption sur ces coagulants synthétiques.

Pour l'ensemble des essais dans le cas $\mathrm{du}$ traitement de rejet de coton (Figures 21 et 22), le rendement d'élimination est le même pour les trois coagulants.

Rendement de décoloration en fonction de la concentration des composés synthétiques ajoutés

Pour l'ensemble des essais de rejet de polyester, la comparaison des deux courbes (Figures 23 et 24), nous a permis de conclure que la granulométrie joue un rôle important dans l'adsorption de la matière colorée du rejet de textile, en particulier pour le coagulant synthétique à base de fer/aluminium. Ceci confirme les résultats précédemment trouvés lors des essais sur la DCO.

Pour l'ensemble des essais de rejet de coton (Figures 25 et 26), les résultats montrent que l'aluminium a une bonne efficacité d'élimination du colorant, ceci peut être expliqué par la grande disponibilité des ions d'aluminium suite à leur dissolution intense en solution.

\section{REFERENCES}

Alinsafi A, Khemis M, Pons MN, Leclerc JP, Yaacoubi A, Benhammou A, Nejemeddine A. 2005. Electro coagulation of reactive textile dyes and textile wastewater. Chemical Engineering and processing, 44: 461- 470.

Baghriche O, Djebbar K, Sehili T. 2008. Etude cinétique de l'adsorption d'un colorant cationique (vert de méthyle) sur $\mathrm{du}$ charbon actif en milieu aqueux. Sciences et Technologie, A, B (27) : 5762.

Bell J, Buckleyc A. 2003. treatement textile dye in the araerobic baffled reactor. Water SA, 29: 129-134.

Bennajah B, Gourich B, Essadki AH, Vial CH, Delmas H. 2009. Defluridation of Morocco drinking water by electrocoagulation/ electroflotation in an electrochemical external-loop airlift reactor. Chemical Energineering Journal, 148: 122-131.

Ben Tahar F, Ben Cheikh R, Blais JA. 2004. Decoloration des eaux usées de levureri par adsorption sur charbon. J. Environ. Eng. Sci., 3: 269 - 277.

Bensaid J. 2009. Contribution à la dépollution des eaux usées par électrocoagulation et par adsorption sur des hydroxydes d'aluminium. Thèse d'état, Université MohamedV, Rabat.

Bouras O. 2003. Propriétés adsorbantes d'argiles pontes organophiles Synthèse et caractérisation. Thèse, université de Limoge, Limoge.

Carmona M, Khemis M, Leclerc JP, Lapicque F. 2006. A simple model to predict the removal of oil suspensions from water using the electrocoagulation technique Chemical Energineering Science, 61: 1233-1241.

Drougi P, Asselin M, Brar SK, Benmoussa H, Blais JF. 2007. Electrochemical removal of pollutants from agro industry 
wastewater. Separation and Purification Thecnologie, 61: 301-310.

Heidmann I, Calmano W. 2007. Removol of $\mathrm{Cr}(\mathrm{VI})$ from model wastewater by electrocoagulation with $\mathrm{Fe}$ electrodes. Separation and Purification Thecnologie, 61: 15-41.

Meçabih Z, Kacimi S, Bouchikhi B. 2006. Adsorption des matières organiques des eaux usées urbaines sur la bentonite modifiée par Fe (III), $\mathrm{Al}$ (III) et $\mathrm{Cu}$ (II). Revue des Sciences de l'Eau, 19(1): 2331.

Neff D. 2003. Apport des analogues archéologiques à l'estimation des vitesses moyennes et à l'étude des mécanismes de corrosion à très long terme des aciers non alliés dans les sols, in Sciences Mécaniques pour l'Ingénieur. Thèse, Université de Technologie de Compiègne, $\mathrm{p} 360$.

Parks GA, 1972. Free energies of formation and aqueous solubility of aluminium hydroxides and oxide hydroxides at $25^{\circ} \mathrm{C}$. Amer. Mineralogist, 57: 1163-1189.

Pillre F, Gaey JF, Maison A. 2001. Document pour la médecine de travail, $85: 336$.

Rebhun M, Lurie M. 1993. Control of organic water by coagulation and flocs separation. Wat. Sci. Tech., 27(11): 1-26.

Torrades F, Garcla, Montano J, Garcla Horta JA, Nunez I, Domenechx, Peral J. 2004. Discoloration and mineralisation of homo and hetero- bireactive dyes under ferton and photo - fenton conditions. Coloration Technol., 120: 188-194.
Zidane F, Berrada B, Lekhlif B, Lounes M, Blais JF. 2006. Performances D'un biofiltre à garnissage plastique pour le traitement d'effluents fortement contaminées en phénol, cadmium et chrome. Journal Environnemental Science, 5: 317-327.

Zidane F, Drogui D, Lekhlif B , Bensaid J, Blais JF, Belcadi S, El kacemi K. 2008. Discoloration of dye-containing effluent using mineral composé synthétiques produced by electrocoagulation, Journal of Hazardous Materials, 155: 153-163.

Zongo I. 2009. Etude expérimentale et théorique du procédé d'électrocoagulation: application au traitement de deux effluents textiles et d'un effluent simulé de tannerie. Thèse d'université de Nancy, Nancy.

Zongo I, Maiga AH, Wéthé J. Valentine G, Leclerc JP, Paternotte G, Lapicque F. 2009. electrocoagulation for the treatment of textile wastewater with $\mathrm{Al}$ or $\mathrm{Fe}$ electodes compared variation of COD levels, turbidity and absorbance. Journal of Hazardous Material, 169: 70-76.

Zuo Q, Chen X, Li W, Chen G. 2008. Combined electocoagulation and electroflotation for removal of fluoride from drinking water. Journal of Hazardous Material, 159: 452-457. 